\title{
Article \\ Population Genomics of Megalobrama Provides Insights into Evolutionary History and Dietary Adaptation
}

\author{
Jing Chen ${ }^{1,2} \oplus$, Han Liu ${ }^{1,2, *}$, Ravi Gooneratne ${ }^{3}$, Yao Wang ${ }^{1,2}$ and Weimin Wang ${ }^{1,2, *} \mathbb{C}$ \\ 1 Key Lab of Agricultural Animal Genetics, Breeding and Reproduction of Ministry of Education, \\ College of Fisheries, Huazhong Agricultural University, Wuhan 430070, China; cjing24511@163.com (J.C.); \\ othnielartorious@gmail.com (Y.W.) \\ 2 Key Lab of Freshwater Animal Breeding, Ministry of Agriculture and Rural Affairs, College of Fisheries, \\ Huazhong Agricultural University, Wuhan 430070, China \\ 3 Faculty of Agriculture and Life Sciences, Lincoln University, Lincoln 7647, New Zealand; \\ ravi.gooneratne@lincoln.ac.nz \\ * Correspondence: liuhan@mail.hzau.edu.cn (H.L.); wangwm@mail.hzau.edu.cn (W.W.)
}

Citation: Chen, J.; Liu, H.; Gooneratne, R.; Wang, Y.; Wang, W. Population Genomics of Megalobrama Provides Insights into Evolutionary History and Dietary Adaptation. Biology 2022, 11, 186. https:// doi.org/10.3390/biology11020186

Academic Editors: Baofeng Su, Rex Dunham and Xu Wang

Received: 4 October 2021

Accepted: 20 January 2022

Published: 25 January 2022

Publisher's Note: MDPI stays neutral with regard to jurisdictional claims in published maps and institutional affiliations.

Copyright: (C) 2022 by the authors. Licensee MDPI, Basel, Switzerland. This article is an open access article distributed under the terms and conditions of the Creative Commons Attribution (CC BY) license (https:// creativecommons.org/licenses/by/ $4.0 /)$.
Simple Summary: Megalobrama is the economically most important freshwater fish genus in China. In recent years, germplasm resources of Megalobrama have been depleting as a result of environmental degradation and artificial factors. In this study, we established the whole genome database of Megalobrama populations using the whole genome re-sequencing technology, explored population genetic structure, and inferred comprehensive evolutionary relationships using principal component analysis and population structure analysis. Furthermore, employing selective sweep analysis, we identified candidate genes related to variations in feeding habits, revealing the molecular mechanisms of environmental adaptability in Megalobrama populations. Taken together, this study describes the population history and genetic diversity of Megalobrama populations and also the molecular mechanisms likely involved their environmental adaptability. These findings will make a substantial contribution to conservation and utilization of Megalobrama germplasm resources.

Abstract: Megalobrama, a genus of cyprinid fish, is an economically important freshwater fish widely distributed in major waters of China. Here, we report the genome resequencing of 180 Megalobrama fish including M. amblycephala, M. skolkovii, M. hoffmanni, and M. pellegrini. Population structure indicated that geographically divergent Megalobrama populations were separated into six subgroups. A phylogenetic tree showed that $M$. skolkovii was more closely related to M. pellegrini than other species and M. hoffmanni was clustered apart from other Megalobrama species, showing a high nucleotide diversity in geographic groups. Treemix validated gene flow from M. amblycephala to M. skolkovii, suggesting that introgression may provide an important source of genetic variation in the M. skolkovii populations. According to the demographic history analysis, it is speculated that Megalobrama might have been originally distributed in the Pearl River with some spread to Hainan Island and northern China due to lower sea levels during the glacial period. Whole-genome selective sweeps analysis demonstrated that M. amblycephala likely developed an enhanced energy metabolism mostly through fatty acid degradation pathways whereas M. hoffmanni possibly regulate lipid absorption via the cholesterol metabolism pathway. Taken together, this study provides a valuable genomic resource for future genetic investigations aiming to improve genome-assisted breeding of Megalobrama species.

Keywords: genome resequencing; Megalobrama; population structure; demographic history; feeding habits

\section{Introduction}

Megalobrama is a cyprinid fish genus that belongs to the subfamily Cultrinae (Cypriniformes, Cyprinidae). It is one of the economically most important fish species, as well as the main aquaculture species in China. The genus Megalobrama contains four recognized 
species, M. amblycephala, M. skolkovii, M. hoffmanni, and M. pellegrini [1]. Previous studies categorized the four species mainly based on morphologic traits and geographic distribution. For instance, M. amblycephala is distinguished by the morphology of the upper orbital bone and oral fissure, and also the caudal peduncle depth/length ratio [2]. It is mainly distributed in large- and medium-sized lakes in the middle and lower branches of the Yangtze River [3]. M. skolkovii differs from the other three species in the longer length of the first swim bladder [2]. It inhabits the Amur, Yangtze, Yellow, and Minjiang rivers [4]. M. hoffmanni has pigment deposits on the scale base that form dark spots on its body surface and exists mostly in the Pearl River and Hainan Island [5]. M. pellegrini dwells in the middle and upper reaches of the Yangtze River and has a unique upper jaw and upper orbital bone shape [6]. Although previous studies have reported biological differences between Megalobrama species, the phylogenetic relationships among M. amblycephala, M. skolkovii, M. hoffmanni, and M. pellegrini remain unknown $[7,8]$.

Four Megalobrama species have distinct feeding behaviors. For instance, M. hoffmanni is an omnivorous fish that feeds mostly on benthic creatures such as freshwater shellfish, river clams, organic detritus, and some aquatic plants [9]. M. skolkovii and M. pellegrini are omnivorous as well, however, they primarily feed on aquatic plants [10]. Importantly, in M. pellegrini, the development of the upper and lower jaws as well as the thickening of the stratum corneum have strengthened the scraping function, making it more conducive to feeding on stationary organisms [6]. M. amblycephala lives in aquatic plant-rich lakes and its diet consists primarily of aquatic plants, emergent plants, floating-leaf plants, and submerged plants. It is classified as herbivorous fish that mainly feeds on aquatic vascular plants [11]. Obviously, Megalobrama species must have evolved metabolic mechanisms that allow them to adapt to varying dietary compositions. However, it is not clear how Megalobrama species regulate carbohydrate, lipid, and protein metabolism to maintain energy balance in the body.

Megalobrama germplasm resources have been rapidly depleting in recent years as a result of many factors, including environmental degradation factors. There are a few reports on Megalobrama genetic resource conservation and population genetics [1,2,4]. By using population genomics, the genetic information of species diversity can be established with the advent of high-throughput sequencing [12,13]. For instance, whole genome re-sequencing has been used to investigate the phylogenetic relationships among differentiated species [14,15]. Using the whole genome of M. amblycephala as reference genome, this study was designed with the following objectives: (1) establish the whole genome database of Megalobrama population using the whole genome re-sequencing technology; (2) explore the genetic structure of populations and infer detailed evolutionary relationships by phylogenetic tree, principal component analysis (PCA), and admixture analysis; (3) illustrate the demographic history of Megalobrama species by pairwise sequentially Markovian coalescent (PSMC) method; (4) determine candidate genes related to variations in feeding habits utilizing selective sweeps analysis that would indicate the molecular mechanism (s) of environmental adaptability of Megalobrama populations. This study will ascertain the genetic diversity, population history, and improve the understanding of the evolution of Megalobrama populations and their molecular mechanisms of environmental adaptability. These findings will represent a significant contribution to conservation and utilization of Megalobrama's germplasm resources.

\section{Materials and Methods}

\subsection{Samples Information}

A total of 180 samples, eight populations of $M$. amblycephala from Liangzi Lake (LZL; $n=12$ ), Poyang Lake (PYL; $n=12)$, Yuni Lake (YNL; $n=12)$, Tiane Island (TEL; $n=12$ ), Dongting Lake (DTL; $n=11$ ), Jinsha River Reservoir of Hongan County (JS; $n=1)$, Shaoguan (SG; $n=2$ ), Yingde (YD; $n=7$ ); six populations of M. skolkovii from Fuyuan (FY; $n=12$ ), JS ( $n=12)$, Qiantang River (QT; $n=12)$, SG $(n=10)$ and YD $(n=5)$, DTL $(n=1)$; four populations of $M$. hoffmanni from Qingyuan (QY; $n=12)$, Zhaoqing (ZQ; $n=12)$, Fengkai 
(FK; $n=12)$, and Hainan (HN; $n=12)$, and one population of M. pellegrini from Longxi River (LX; $n=12$ ) were collected. The fin tissues from all samples were preserved in $95 \%$ ethanol and stored at $-80{ }^{\circ} \mathrm{C}$.

\subsection{Genome Sequencing and Sequence Alignment}

Genomic DNA was extracted from the fin of each fish using a DNA extraction kit (Tiangen, Beijing, China). Paired-end sequencing libraries with an insert size of approximately 300-400 bp were constructed for each individual using the BGISEQ 500 platform and sequenced on an MGISEQ-2000 sequencer. After removing joint pollution and lowquality reads, the filtered high-quality clean data were for subsequent comparative analysis. The filtering was performed using SOAPnuke (v1.5.6) (http:/ / github.com/BGI-flexlab/ SOAPnuke (accessed on 9 September 2020)) software. The specific filtering conditions were as follows: removed reads containing adapter, low-quality reads (base quality value less than or equal to 15 bases account for $10 \%$ ), and reads with $\mathrm{N}$ bases greater than $10 \%$. All cleaned reads were mapped to the $M$. amblycephala reference genome (mixed assembly of second and third generations) using BWA-MEM (0.7.13-r1126) with default parameters [16] The effective depth was approximately $16.98 \times$ per individual (Table S1). The Samtools v1.9 software was used to convert the SAM format to BAM format [17]. The SortSam.jar tool of Picard v1.117 software was used to compare and sort the files.

\subsection{Discovery of Genomic Variations}

The variation detection was performed with the Genome Analysis Toolkit (GATK, version v3.3.0) with the UnifiedGenotyper method [18]. Single nucleotide polymorphisms (SNPs) were filtered both per population and per individual using GATK hard filtering [19] with the following stringent filtering criteria: $\mathrm{QD}<2.0$ । । FS $>60.0$ । | MQ $<40.0$ । । ReadPosRankSum $<-8.0$ । | MQRankSum $<-12.5$. Insertions and deletions (Indels) were filtered with parameters: QD $<2.0$ | | FS $>200.0$ | | ReadPosRankSum $<-20.0$. SNPs were pruned for minor allele frequency (MAF) $>1 \%$ in each population. The snpEff (v4.3T) software was used to annotate SNPs and count the annotation information [20].

\subsection{Population Structure Analysis and Species Definition Analysis}

The principal component analysis (PCA) was performed using EIGENSOFT (version 4.2) and the significance level of eigenvectors was determined with the Tracy-Widom test [21]. Admixture version 1.3.0 was used to analyze the population structure [22] 50 different random seeds were selected and indifferently analyzed $K$, and CLUMPP (v1.1.2) software was used to merge 50 analytical results [23]. DISTRUCT (v1.1) software was used to draw the population structure diagram [24]. Population structure was inferred by admixture with 10-fold cross-validation. Admixture was run for each possible group number ( $K=2$ to 8 ) with 200 bootstrap replicates. No admixed individuals were used to construct the phylogenetic tree by FastTree v2.1 software [25].

\subsection{Linkage Disequilibrium and Diversity Analysis}

Linkage disequilibrium (LD) is the non-random association between alleles of different loci in the population and $r^{2}$ can be used to calculate the degree of linkage disequilibrium between two markers. In this study, the software PopLDdecay (v3.40) (https://github. com/BGI-shenzhen/PopLDdecay (accessed on 4 December 2020)) was used to set the parameters (-MaxDist 1000 -MAF 0.05 -Miss 0.1) to perform linkage disequilibrium analysis on SNPs [26]. LD is mostly affected by recombination, artificial selection, and population type. Generally, the distance at which each population decays to half of the maximum $\mathrm{r}^{2}$ value is used as the decay distance of the population. Based on all credible SNP data, vcftools (v0.1.13) software was used to calculate the nucleotides polymorphisms $(\pi)$ of the four Megalobrama species and the differentiation index (Fst) between species [27]. 


\subsection{Gene Flow Analysis}

Treemix software was used to investigate the gene flow between populations [28]. The analysis utilized allele frequencies to construct a maximum likelihood tree and infer the migration events between populations. It is assumed that there are $n$ migration events, and each migration event is calculated separately. Two analyses were performed. In one, the different Megalobrama populations were divided into four subgroups: M. hoffmanni, M. skolkovii, M. amblycephala, and M. pellegrini, with M. hoffmanni as the out group. In the other, $M$. hoffmanni was recognized as an outgroup with nine subgroups including M. pellegrini from Longxi River; M. skolkovii from Fuyuan, Shaoguan, Qiantang River, and Jinsha River Reservoir; and M. amblycephala from Dongting Lake, Poyang Lake, and Liangzi Lake added for gene flow analysis.

\subsection{Reconstruction of the Ancestral Geographic Distribution of Megalobrama}

Based on the phylogenetic tree generated by the credible SNPs, we used the statistical dispersal-vicariance analysis (S-DIVA), dispersal-extinction-cladogenesis (DEC), and Bayesian binary MCMC (BBM) models of reconstruct ancestral state in phylogenies (RASP) 4.0 software to reconstruct the geographical distribution of Megalobrama ancestors [29,30]. In DEC, the possibility of gene exchange between non-adjacent distribution areas was limited to zero. In BBM, the MCMC chain was run for 10 million generations. The sampling frequency was 1000 and the first $25 \%$ samples were discarded as burn-in. The number of the most allowed distribution locations was set to 4 . In the final analysis, the outgroup information was deleted. The division of the distribution area was mainly based on the Chinese water systems.

\subsection{Demographic History Reconstruction and Estimates of the Divergence Time}

The pairwise sequentially Markovian coalescent (PSMC) method was used to model the history of four Megalobrama species (at least $20 \times$ ) and infer the historical changes in their effective population sizes and population separations. The PSMC model was utilized to estimate changes in the effective population size using heterozygous sites across the genome [31]. The number of free atomic time intervals (-p option) was reset with the initial value of $r=\theta / \rho$ (-r option) according to previous research [31]. Based on the estimates from the M. amblycephala genome, an average mutation rate $(\mu)$ of $0.1 \times 10^{-8}$ per base per generation and a generation time $(\mathrm{g})$ of 2.5 years were used for analysis.

\subsection{Detection SNPs under Selective Sweep Analysis}

To investigate the potential selective signals during M. amblycephala and M. hoffmanni dietary adaptation, genomic regions were scanned based on genome-wide calculations for selective sweeps by differentiation index (Fst), nucleotide polymorphism ratio (PiR), and extended haplotype homozygosity between populations (XP-EHH). vcftools software [27] was used to analyze the Fst and PiR values with a sliding window of $50 \mathrm{~Kb}$ and a step size of $10 \mathrm{~Kb}$. For XP-EHH analysis, selscan v1.2.0 software [32] was applied and norm parameter used to normalize the original XP-EHH value, and the average value in the sliding window calculated according to the $50 \mathrm{~Kb}$ sliding window and $10 \mathrm{~Kb}$ step size after the standardized value. The common regions of Top 5\% Fst, High/Low top 5\% PiR, and High/Low top $5 \%$ XP-EHH were identified as the selected regions. For the M. amblycephala populations, 132 regions with a total length of $6.68 \mathrm{M}$ contained 166 genes were identified from the comparison between M. amblycephala and M. skolkovii; 133 regions with a total length of $6.67 \mathrm{M}$ containing 179 genes identified from the comparisons between M. amblycephala and M. pellegrini; 97 regions with a total length of $5.32 \mathrm{M}$ containing 191 genes identified from the comparisons between M. amblycephala and M. hoffmanni. For the M. hoffmanni populations, 75 regions with a total length of $4.14 \mathrm{M}$ containing 126 genes were identified from the comparisons between M. amblycephala and M. hoffmanni. Genes from these selective regions were subjected to a functional enrichment analysis of selected genes with Gene Ontology (GO) and Kyoto Encyclopedia of Genes and Genomes (KEGG) (DAVID, v6.8) [33]. 
Statistical significance was accessed by using a modified Fisher's exact test and Benjamini correction for multiple testing. The calculated $p$-value was based on Bonferroni Correction and the functional terms or pathways with corrected $p$-value $<0.05$ defined as significantly enriched functional terms.

\subsection{RNA Isolation and Real-Time qPCR}

In this study, the liver and spleen tissues from M. amblycephala in LZL and M. hoffmanni in QY populations were selected. The total RNA of these samples was extracted by Trizol reagent (Invitrogen, Carlsbad, CA, USA). The quality and quantity of RNA were detected by NanoDrop 2000 (Thermo Scientific, Waltham, MA, USA), agarose gel electrophoresis and a UV spectrophotometer (Thermo Fisher Scientific Inc., Waltham, MA, USA). Then $1 \mu \mathrm{g}$ total RNA was reverse-transcribed into cDNA using cDNA Synthesis Kit (TaKaRa, Shiga, Japan). qPCR primers are listed in Table S2. We screened and compared three reference genes ( $\beta$-actin, Gapdh, and 18S rRNA), and found that $\beta$-actin was more stable than other reference genes (Figure S1 and Table S3). Thus, $\beta$-actin was used as an internal control. The expression pattern of genes was investigated by the real-time qPCR method with a quantitative thermal cycler (MyiQTM 2 Two Color Quantitative PCR Detection System, Bio-Rad, Hercules, CA, USA). Calculations for relative expression were conducted by the $2^{-\Delta \Delta C t}$ method. Statistical analysis was performed with SPSS 16.0 software and results are reported as mean \pm standard error (SE). The data were analyzed by independent sample $t$-test, and a $p$-value $<0.05$ was considered to indicate a statistical significance.

\section{Results}

\subsection{Genome Resequencing and Variation Calling}

A total of 180 Megalobrama fish representing different geographical populations in China were selected for genome resequencing (Figure 1, Table S4). To explore the phylogenetic relationships and evolutionary history of Megalobrama, all samples were sequenced to an average sequencing depth of $\sim 17.43 \times$ per individual yielding $94 \%$ sequencing coverage, an average Q20 of 97\% and an average Q30 of 90\% (Table S5). After filtering and quality control, the sequencing generated a total of $4.063 \mathrm{~Tb}$ raw databases and $3.483 \mathrm{~Tb}$ clean databases. We mapped all individuals to the $M$. amblycephala genome and detected $31,857,189$ SNPs in the Megalobrama populations (Table S6). 


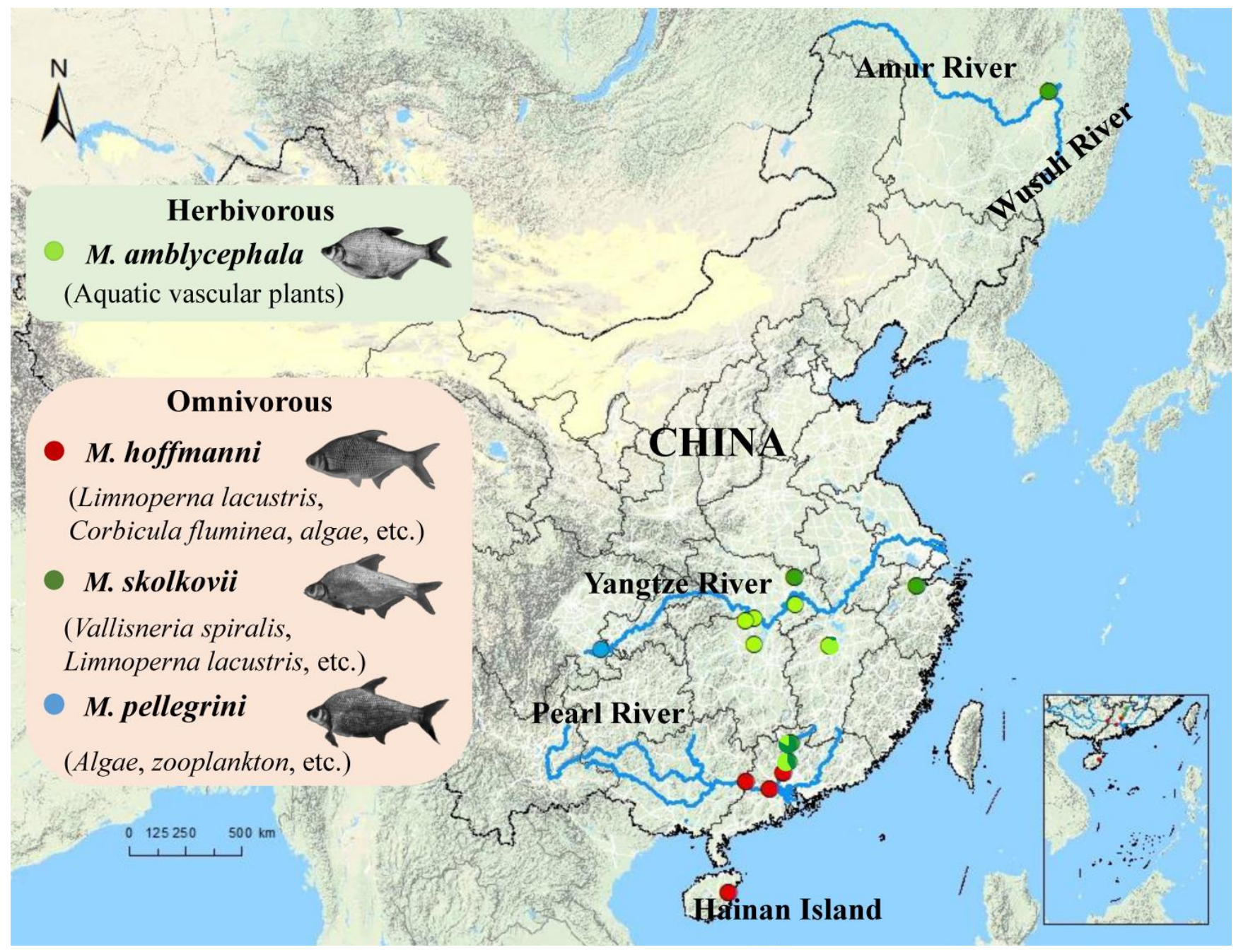

Figure 1. Geographic map indicating the distribution and feeding habits of the Megalobrama species in this study. Circles reflect the geographic regions where the samples were collected from. The blue lines represent the rivers.

\subsection{Phylogeny and Population Structure Analysis}

Admixture analysis was used to cluster individuals based on all high-confidence SNP sites. When ancestry components $(K)=6$, six geographically distributed ancestral components $(K)$ were labeled as: HN and Pearl River M. hoffmanni populations; M. amblycephala populations; FY and other M. skolkovii populations; and M. pellegrini populations (Figure 2A). This finding was supported by cross-validation (when $K=6$, the cross-validation error was minimum) and was consistent with the phylogeny and biogeographic distribution (Figure 2B). Using the Danio rerio as an outgroup, a phylogenetic tree was constructed by the maximum likelihood method based on all non-admixed individuals. All of the samples were clustered into three branches. Initially, M. amblycephala and $M$. hoffmanni were clustered into a single branch and M. skolkovii and M. pellegrini clustered into one branch (Figure 2C). The findings revealed a close genetic affinity between $M$. skolkovii and M. pellegrini groups whereas the M. hoffmanni population showed genetic divergence from the other Megalobrama groups. Additionally, principal components analysis (PCA) provided supporting evidence for these groupings (Figure 2D). The first four principal components divided the Megalobrama populations into six subgroups. Taken together, the results provided compelling evidence that Megalobrama species from various geographical distributions can be divided into six different subgroups. 
A

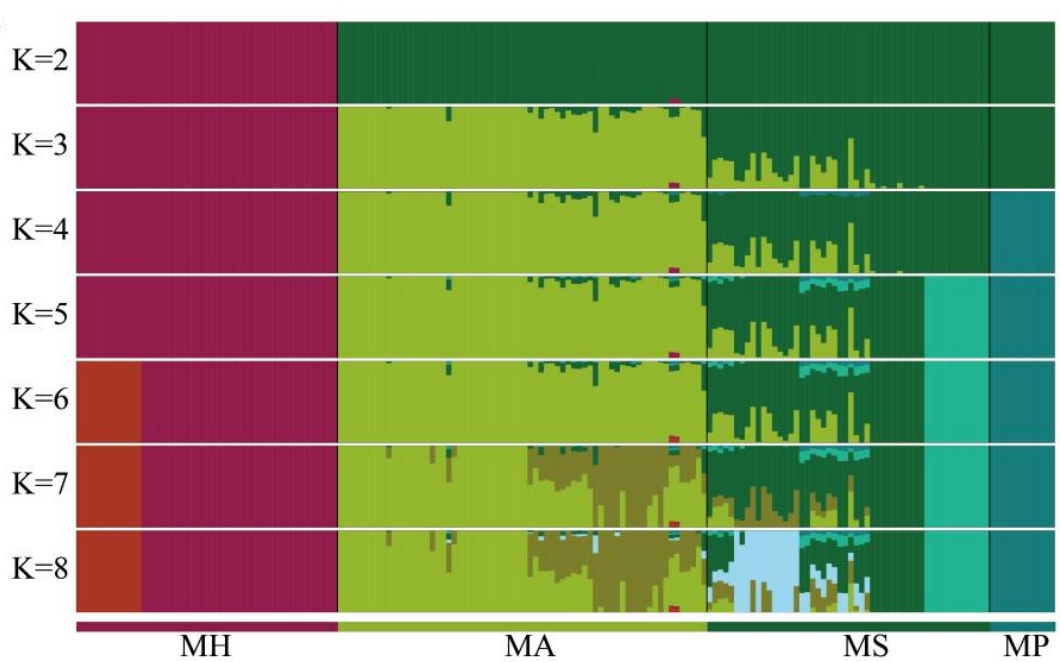

B

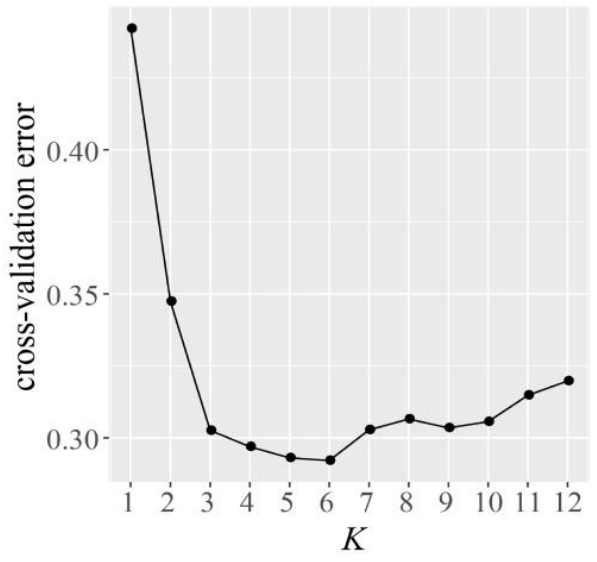

$\mathrm{C}$

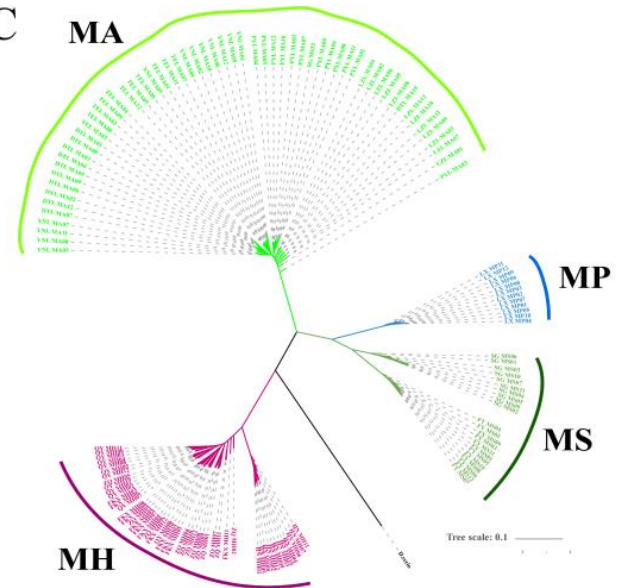

$\mathrm{D}$

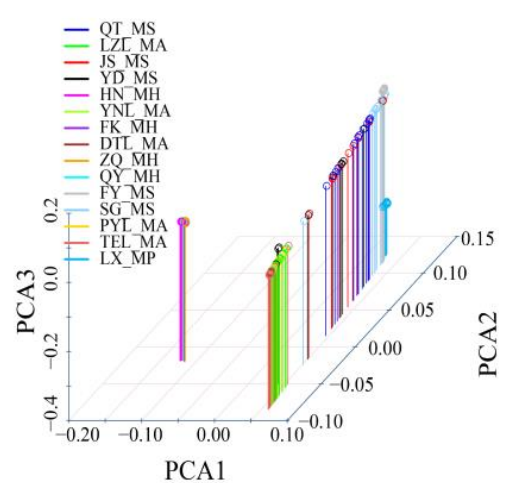

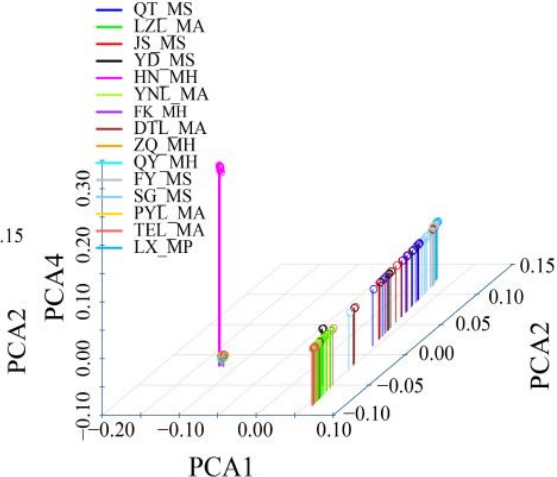

Figure 2. Phylogenetic analysis of different Megalobrama geographical populations. (A) Population genetic structure of 180 Megalobrama fish. The length of each colored segment represents the proportion of the individual genome inferred from ancestral populations $(K=2-8)$. The species name is at the bottom. (B) Cross-validation (CV) error for varying values of $K$ in the admixture analysis. The minimum of estimated CV error on $K=6$ suggests the most suitable number of ancestral populations. (C) Maximum-likelihood-based phylogenetic tree constructed using no admixed individuals. The scale bar represents pairwise distances between different individuals. Different colors represent different Megalobrama species. (D) Principal component analysis (PCA) of Megalobrama populations. Eigenvector 1, 2, 3, and 4 explained $45.06 \%, 18.03 \%, 4.00 \%$, and $1.73 \%$ of the total variance, respectively. MH refers to M. hoffmanni, MA to M. amblycephala, MS to M. skolkovii, MP to M. pellegrini.

\subsection{M. amblycephala Introgression into M. skolkovii}

In this study, we used Treemix to confirm the gene flow from M. amblycephala to M. skolkovii. Using M. hoffmanni as the outgroup, among the four Megalobrama species, only one migration event was inferred from the M. amblycephala to the M. skolkovii with approximately $50.77 \%$ DNA gene flow from M. amblycephala to M. skolkovii (Figure 3A). Moreover, the migration patterns of these two species' geographic populations demonstrated that LZL, DTL, TEL, YNL, and PYL M. amblycephala populations introgressed into the FY, SG, QT, and JS M. skolkovii populations. Notably, the migration weight of $M$. amblycephala introgressed into QT and JS populations was greater than that of the FY and SG populations (Figure S2). 
A

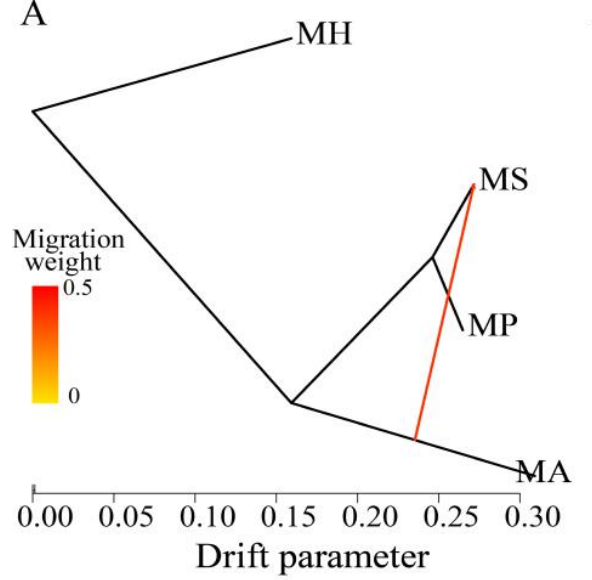

B

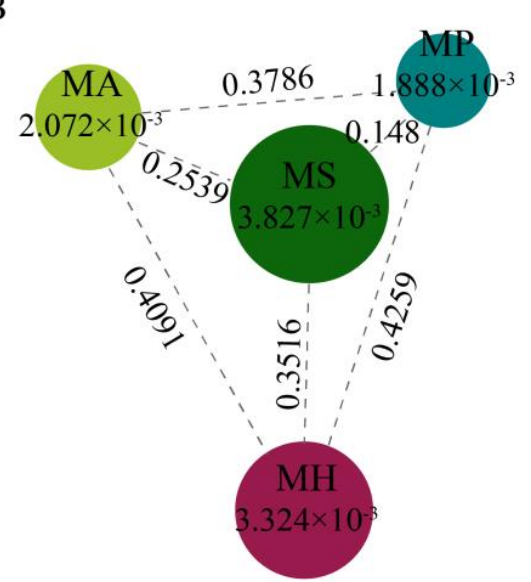

C

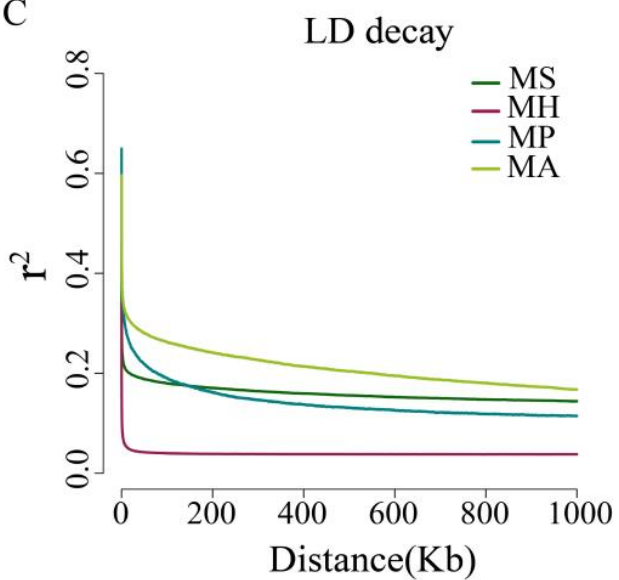

Figure 3. Population genetic analysis of Megalobrama species. (A) Gene flow analysis of Megalobrama species. Arrows indicate migration events that occur between populations. The heat map indicates migration weight. (B) Nucleotide polymorphism $(\pi)$, and differentiation index (Fst) of the four Megalobrama species. The largest circle represents the large $\pi$ value, and the longer line segment represents the large Fst value. MH refers to M. hoffmanni, MA to M. amblycephala, MS to M. skolkovii, MP to M. pellegrini. (C) Decay of linkage disequilibrium (LD) patterns for the four Megalobrama species inferred by the phylogenetic trees.

\subsection{Linkage Disequilibrium and Genetic Diversity}

The genetic diversity $(\pi)$ of $M$. skolkovii and M. hoffmanni were estimated to be $3.827 \times 10^{-3}$ and $3.324 \times 10^{-3}$, respectively, which was relatively high in comparison to M. amblycephala $\left(2.072 \times 10^{-3}\right)$ and M. pellegrini $\left(1.888 \times 10^{-3}\right)$. The low genetic differentiation (Fst) between M. skolkovii and M. pellegrini (0.148), and the high Fst between M. hoffmanni and M. amblycephala (0.4091), M. skolkovii (0.3516), and M. pellegrini (0.4259) (Figure 3B) are consistent with the phylogeny analysis. Between the four Megalobrama species, linkage disequilibrium (LD) and correlation coefficient $\left(r^{2}\right)$ values were calculated. The decay of LD reached half the maximum average $r^{2}$ at a distance of $24 \mathrm{~Kb}, 4 \mathrm{~Kb}, 1.8 \mathrm{~Kb}$, and $0.2 \mathrm{~Kb}$ for M. amblycephala, M. pellegrini, M. skolkovii, and M. hoffmanni, respectively (Figure 3C). Therefore, M. skolkovii and M. hoffmanni displayed a faster LD decay rate than M. amblycephala and M. pellegrini.

\subsection{Demographic History of Megalobarma Species and Species Delimitation}

The split times based on the relative cross-coalescent rates (RCCR) among the four Megalobrama species reached 0.5 suggesting a split between $M$. hoffmanni and other species 3-5 Mya. The cross-coalescence analysis suggested a decline to 0.5 between $M$. amblycephala and M. skolkovii or M. pellegrini at $\sim 1.3 \mathrm{Mya}$, and a decline to 0.5 between M. skolkovii and M. pellegrini around 0.3-0.4 Mya (Figure 4A). The PSMC method used to reconstruct the demographic history of six Megalobrama subgroups indicated that the effective population size ( $\mathrm{Ne}$ ) peak of M. amblycephala and M. pellegrini was 2.5 Mya and 4 Mya, respectively, compared to 3-4 Mya of the Ne peak for the M. skolkovii subgroups. After that, the FY M. skolkovii subgroup continued to shrink, whilst the Ne curves of other M. skolkovii subgroup split at 0.2 Mya expanded (Figure 4B). Moreover, the two Ne peaks of M. hoffmanni occurred at $0.3 \mathrm{Mya}$ and $5 \mathrm{Mya}$, respectively, during the middle pleistocene geological period (Figure 4C). Interestingly, the split of Ne curves of the two M. hoffmanni subgroups occurred about $0.4 \mathrm{Mya}$, indicating a population divergence at this time. The ancestral reconstruction analysis revealed two different biogeographic evolutionary processes to investigate the ancestral distribution of genus Megalobrama. According to the BBM analysis, the genus Megalobrama was originally distributed in the Pearl River and then spread to Hainan Island and Northern China. However, the analysis based on the S-DIVA and DEC 
models showed that the Megalobrama ancestors originally inhabited the Pearl River and Yangtze River, before spreading to the Amur and Wusuli Rivers (Figure S3).

A

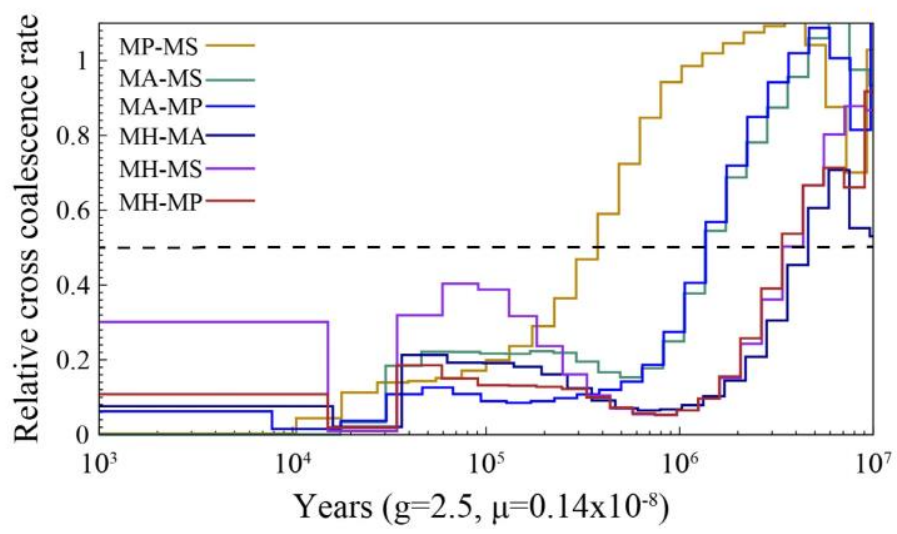

B

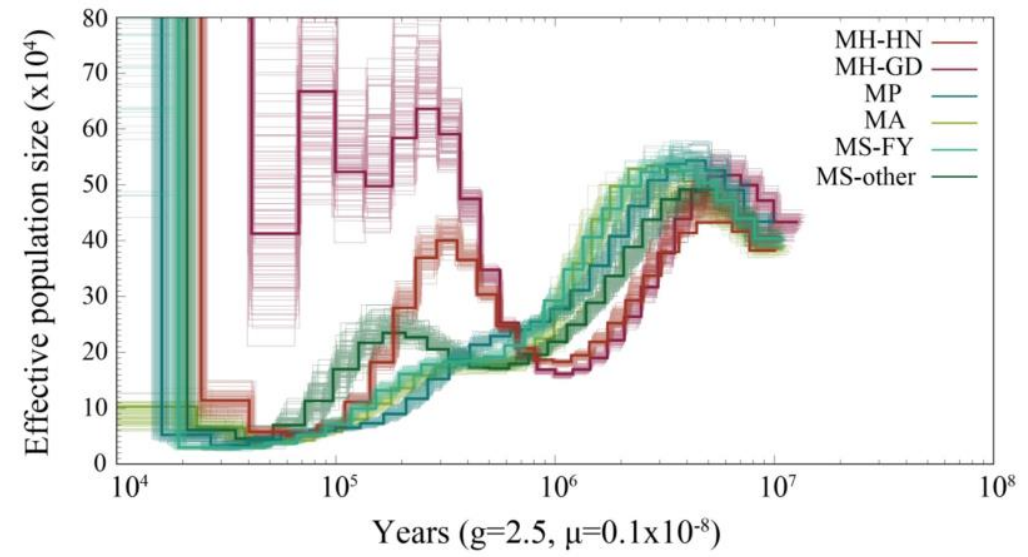

$\mathrm{C}$

Pliocene epoch

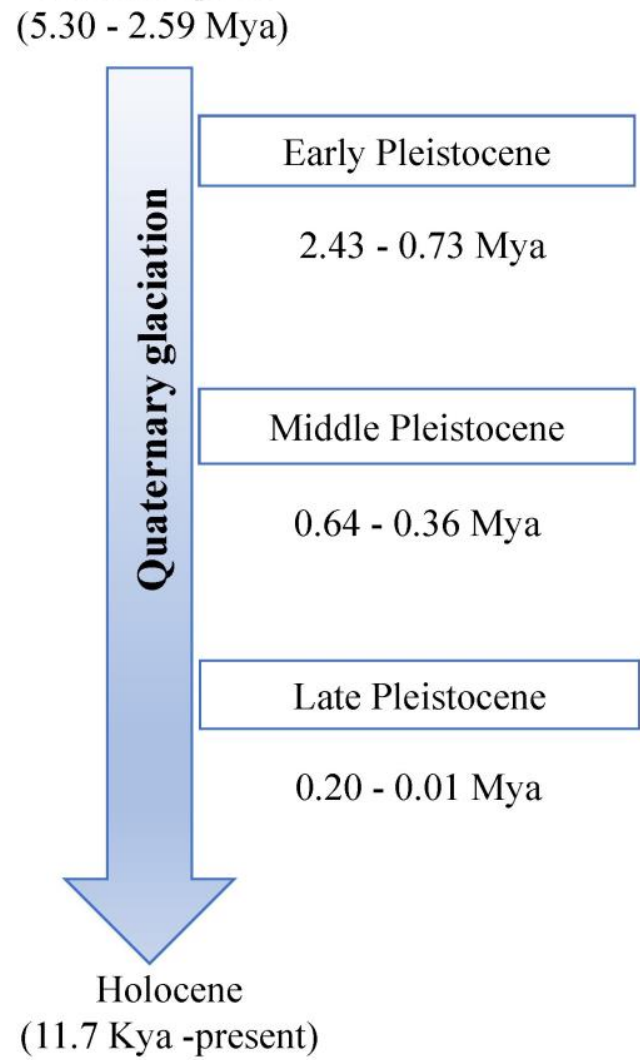

Figure 4. Demographic history of Megalobrama species. (A) Relative cross coalescence rates (CCR) between Megalobrama populations. When the two populations are completely mixed, the CCR is close to one. When they are completely split, the CCR is close to zero. The dotted line indicates that the CCR is 0.5. MP, MS, MA, and MH refer to M. pellegrini, M. skolkovii, M. amblycephala, and M. hoffmanni. $g$ (generation time $)=2.5$ years; $\mu$ (neutral mutation rate per generation $)=0.14 \times 10^{-8}$. (B) PSMC model estimates changes in the effective population size over time, representing variation in inferred $\mathrm{Ne}$ dynamics. The undulating broken line in the figure is the estimated effective population size of each population in the evolutionary history. The time axis is not divided into deciles. $\mu=0.1 \times 10^{-8}$. MHHN, MH-GD, MP, MA, MS-FY, and MS-other refer to the Hainan population of M. hoffmanni, Pearl River populations of M. hoffmanni, M. pellegrini, M. amblycephala population, Fuyuan population of $M$. skolkovii, and other M. skolkovii populations. (C) Timeline of Quaternary glaciation. Mya = million years ago.

\subsection{Selective Sweeps for Dietary Adaptation of Megalobrama}

To investigate the potential selective signals during $M$. amblycephala dietary adaptation, we scanned the genomic regions based on genome-wide calculations for selective sweeps by estimating Fst, PiR, and XP-EHH values. Candidate genes were discovered in the common region of top 5\% Fst, High/Low top 5\% PiR, and High/Low top 5\% $\mathrm{XP}-\mathrm{EHH}$, inferred from the comparisons between M. amblycephala and the other three species (Figure 5A, Figures S4 and S5A). Fatty acid degradation, glycerolipid metabolism, beta-alanine metabolism, arginine and proline metabolism, histidine metabolism, insulin secretion, and lysine degradation, among other metabolic processes were associated with a significant portion of the candidate genes, according to GO and KEGG analyses (Figure S6). M. amblycephala mainly feeds on high-fiber and low-energy aquatic vascular plants. The candidate genes of M. amblycephala were enriched in fatty acid degradation and corre- 
A

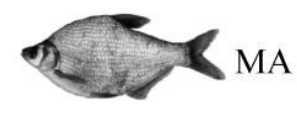

Herbivorous (aquatic vascular plants)

B

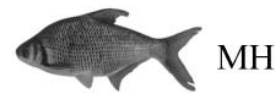

Omnivorous

(benthic animals and a few aquatic plants) sponding upstream and downstream pathways. For instance, Aldh3a2 and Acss3 genes in fatty acid $\alpha$-oxidation, Hadhb gene in fatty acid $\beta$-oxidation, Akt2 gene in insulin signaling pathway, Aldh3a2 gene in glycolysis/gluconeogenesis, Gbe1 gene in starch and sucrose metabolism, Acsbg2 gene in fatty acid biosynthesis, and Aldh3a2 gene in valine, leucine, and isoleucine degradation (Figure 6A and Table S7). Moreover, these genes exhibited a high expression pattern in the liver and/or spleen tissue of M. amblycephala compared with M. hoffmanni (Figure 6B-E,b-e).
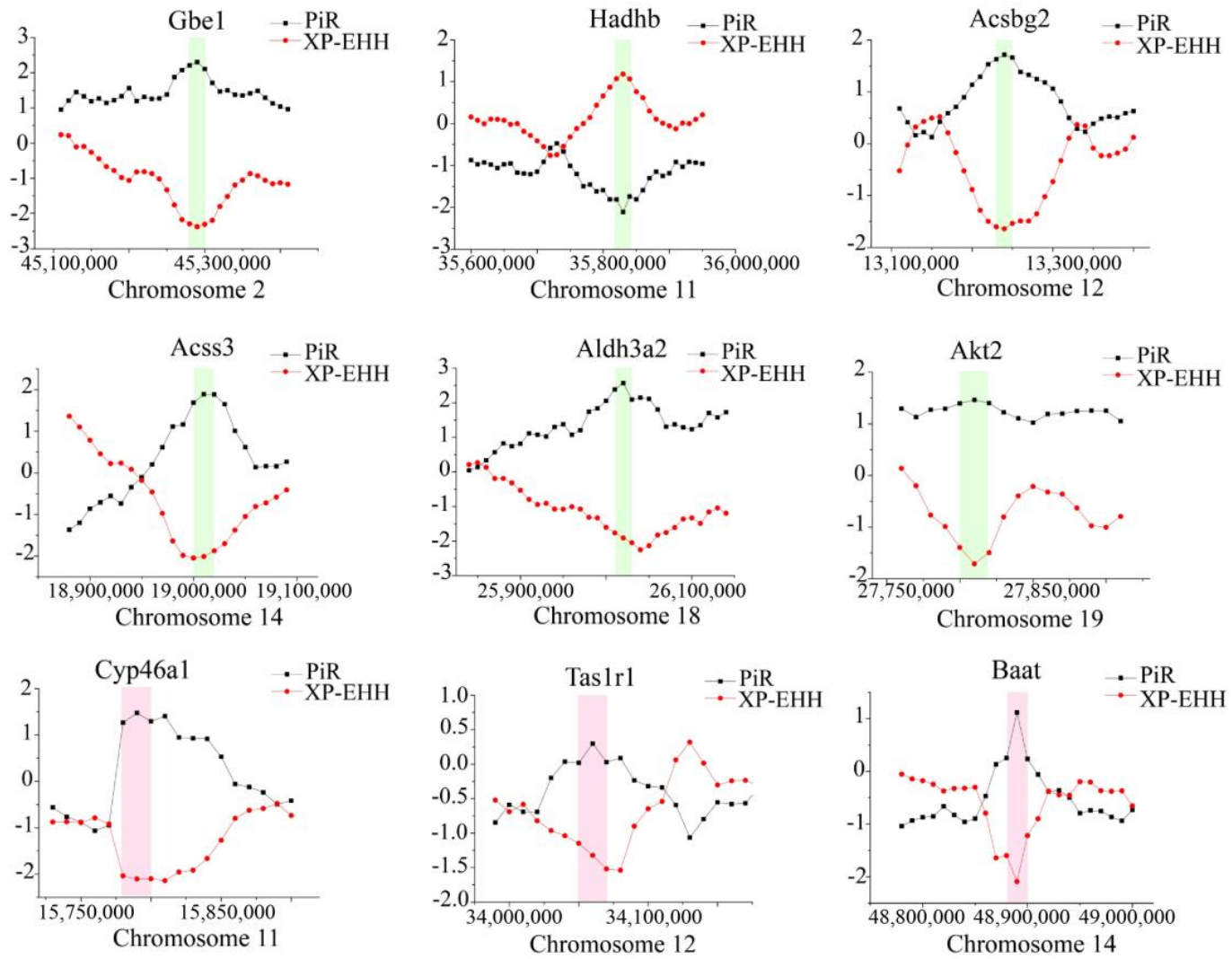

Figure 5. Genome-wide inference of selection sweeps on chromosomes during the diet adaptation of M. amblycephala (A) and M. hoffmanni (B). MA and MH refer to M. amblycephala and M. hoffmanni. Gbe1, Akt2, and Aldh3a2 were identified from the comparison between M. amblycephala and M. skolkovii, Acss3, and Acsbg2 from the comparison between M. amblycephala and M. pellegrini, Hadhb from the comparison between M. amblycephala and M. hoffmanni, and Cyp46a1, Tas1r1, and Baat from the comparison between $M$. hoffmanni and M. amblycephala. The black curve indicates the nucleotide polymorphism ratio (PiR) analysis, and red curve indicates extended haplotype homozygosity between populations (XP-EHH) analysis. The green and pink boxes represent the position of the selected genes on the chromosomes. 
A

Valine, leucine and isoleucine degradation

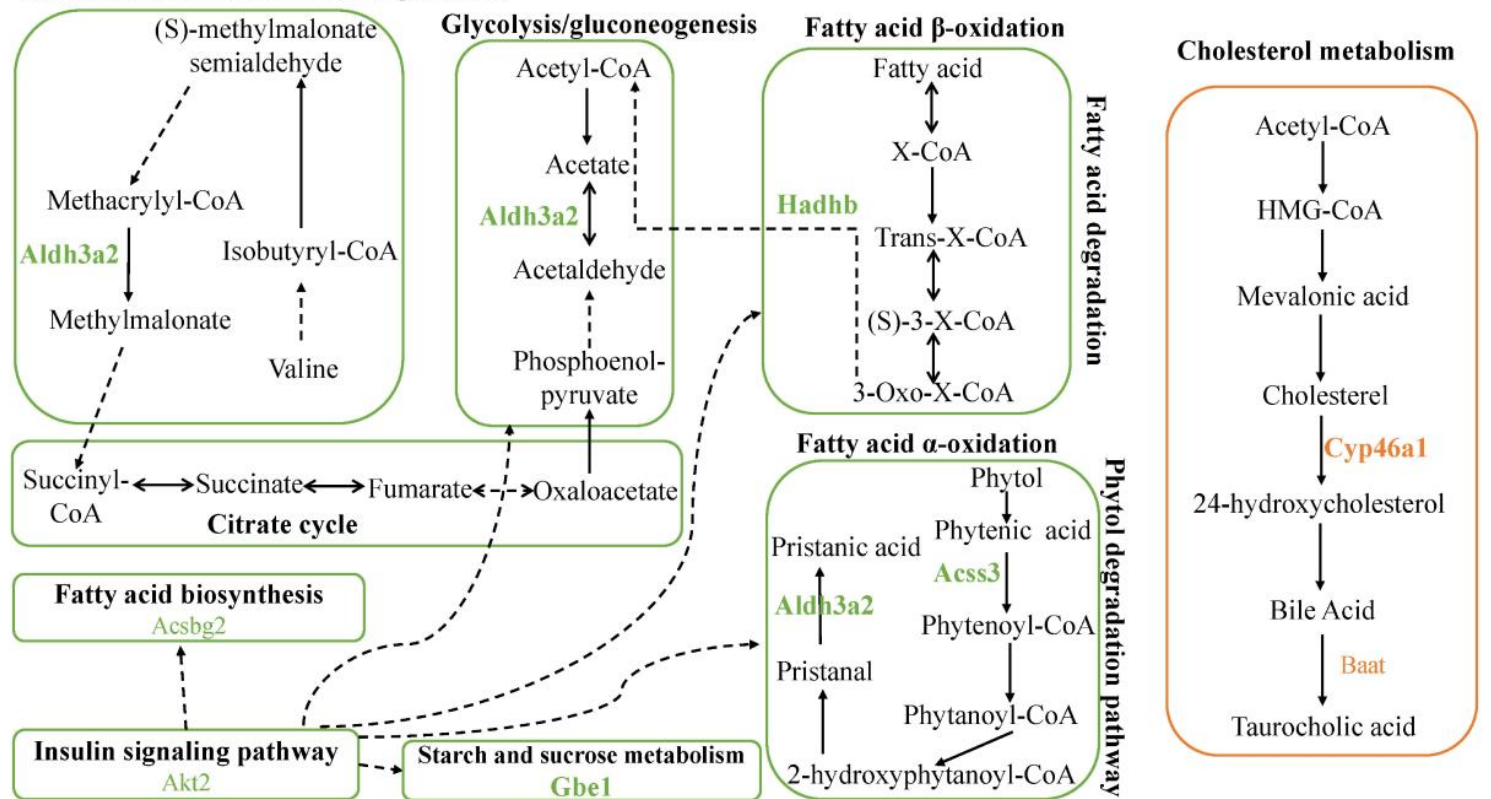

B

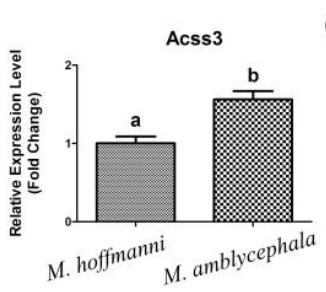

b

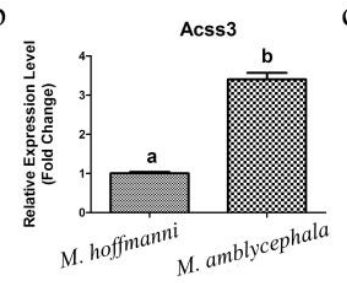

$\mathrm{C}$

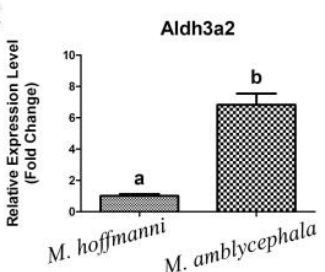

$\mathrm{D}$

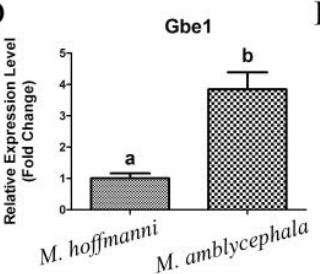

d

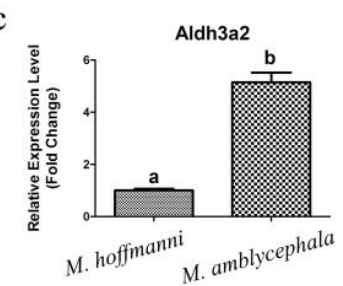

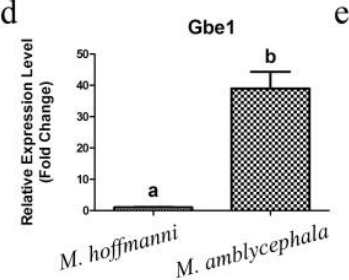

$\mathrm{E}$
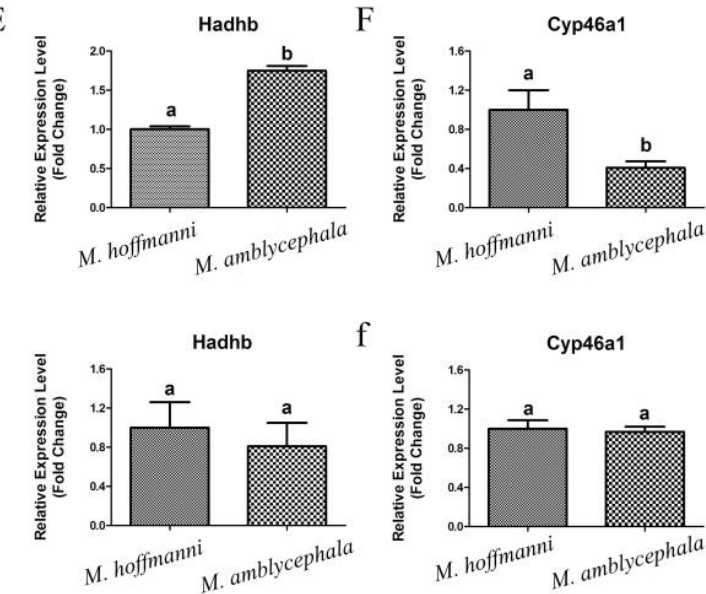

Figure 6. Metabolism pathways and expression pattern of candidate genes. (A) Candidate genes of M. amblycephala and M. hoffmanni enriched in the metabolism pathways. The lines and selected genes in M. amblycephala and M. hoffmanni are indicated in green and pink, respectively. The dashed lines used to connect KEGG pathways represent indirect relationships. (B-F) The expression pattern of candidate genes (Acss3, Aldh3a2, Gbe1, Hadhb, and Cyp46a1) in the liver tissue of M. amblycephala and M. hoffmanni. (b-f) The expression pattern of candidate genes (Acss3, Aldh3a2, Gbe1, Hadhb, and Cyp46a1) in the spleen tissue of M. amblycephala and M. hoffmanni. Different letters indicate a significant difference $(p<0.05)$.

Among the three omnivorous Megalobrama species, the food composition of M. hoffmanni is rich in zoobenthos. The common regions of top 5\% Fst, High top 5\% PiR, and Low top 5\% XP-EHH were the selected regions inferred from a comparison of M. hoffmanni and M. amblycephala, which covered a total of 75 selected regions with a length of $4.14 \mathrm{Mb}$ and including 126 genes in M. hoffmanni. These genes were found to be abundant in cellular and metabolic processes including taste transduction, fatty acid elongation, biosynthesis of unsaturated fatty acids, and biosynthesis of amino acids according to GO and KEGG enrichment analysis (Figure S7). Interestingly, we found some candidate genes (Cyp46a1 and Baat) involved in cholesterol metabolism (Figure 5B, Figure 6A, Figure S5B and Table S7). The results indicated that compared with $M$. amblycephala, the Cyp46a1 gene was highly expressed in the liver of M. hoffmanni, but not in the spleen (Figure 6F,f). Furthermore, 
functional annotation indicated that the umami taste receptor gene Tas1r1 was abundant in the M. hoffmanni sensory system.

\section{Discussion}

Until now, the main resources available to investigate the evolution of Megalobrama species were morphological and mt DNA analyses. There have been some controversies over the Megalobrama species in terms of phylogenetic relationship and species boundaries $[4,7,8]$. Our genomic population datasets revealed that all Megalobrama populations could be separated into six subgroups based on the geographical isolation, with each of M. hoffmanni and M. skolkovii divided into two subgroups. M. skolkovii was more closely related to M. pellegrini than M. amblycephala and M. hoffmanni, and M. hoffmanni clustered apart from other Megalobrama species, showing a high nucleotide diversity and fast LD decay rate in the geographic groups. This finding contributes to resolving the fundamental issue of the phylogenetic relationship of the four Megalobrama species. Recently, artificial introductions have hampered the protection of the Megalobrama genus germplasm resources, particularly M. amblycephala and M. skolkovii. Treemix validated gene flow from M. amblycephala to M. skolkovii. We speculate that, as a result of the introduction of M. amblycephala, introgression increased the nucleotide polymorphism of $M$. skolkovii. It implies that introgression may provide an important source of genetic variation in $M$. skolkovii populations.

The glacial epoch saw a dip in sea-level of 80-130 m compared to the present, wide stretches of the continental shelf emerged and a land bridge formed across the South China Sea [34]. With this connection, the Pearl River M. hoffmanni population likely expanded its distribution range, resulting in the Hainan population. After the glaciers subsided, sea-level rise triggered a phylogeographic break which differentiated the freshwater fish populations living on Hainan Island from those on mainland [35]. It has been reported that the South China Sea transgression during the late Pleistocene interglacial or post-glacial period may have reduced the habitat territory for freshwater fish, resulting in population contraction [36]. Consequently, the effective population size of $M$. hoffmanni in the Pearl River declined throughout this time period. The two critical features of the Quaternary Ice Age are the change of sea level and the existence of high mountains, especially the uplift of the Qinghai-Tibet Plateau, the Yunnan-Guizhou Plateau and the western high mountains, and the large-scale crustal subsidence in the east that has caused gradual disintegration of most water systems [37]. The extant distribution of the freshwater fish fauna in China developed during the elevation of the Qinghai-Tibet Plateau from the late Tertiary to the early Quaternary period [38]. With the uplift of the Qinghai-Tibet Plateau, the original lake basins in the middle and lower reaches of the Yangtze River progressively disintegrated, and the closed lake basins merged, establishing the initial embryonic shape of the Yangtze River. M. pellegrini, M. amblycephala, and M. skolkovii populations in the Yangtze River experienced population contraction due to changes in terrain. However, the increase in the M. skolkovii population in the Qiantang River, adjacent to the East China Sea, may have been caused by a sea-level reduction during the glacial period.

To adapt to their habitat environment, Megalobrama species have evolved and adapted to different food compositions. M. amblycephala, for example, feeds mainly on high-fiber, low-energy aquatic plants. The candidate genes in selective regions of M. amblycephala were associated with lipid metabolism and also the corresponding upstream and downstream pathways. For instance, the Hadhb gene encodes enzymes involved in the final reactions that catalyze the $\beta$-oxidation of fatty acids [39]. Aldh3a2 is a typical fatty aldehyde dehydrogenase that catalyzes the oxidation of long-chain aldehydes produced by lipid metabolism [40]. It has been shown that adaptive alterations in fatty acids $\beta$-oxidation and lipid metabolism can increase the amount of ATP required by the foregut fermenters [41]. Additionally, plant cell walls (such as cellulose and hemicellulose) can be degraded to produce short-chain fatty acids, which are high in energy and serve as the primary source of energy for foregut fermenters [42]. Moreover, Aldh3a2 is implicated in the phytol degradation pathway and plays a dominant role in the oxidation of pristine to pristanic acid [43]. 
Phytol is a side chain of the plant pigment chlorophyll that following consumption by mammals, can be metabolized in the body to form phytanic acid and norphytanoic acid by $\alpha$-oxidation [44]. Phytol and its metabolites are major sources of energy in fish oxidative metabolism. As a result, $M$. amblycephala may have developed a complete fatty acid degradation pathway associated with $\alpha$-oxidation and $\beta$-oxidation of fatty acids to enhance energy metabolism and effectively absorb and utilize scarce nutrients.

The diet of M. hoffmanni consists primarily of benthic animals and little aquatic vegetation. Interestingly, we discovered that the candidate genes in the M. hoffmanni selective regions were associated with cholesterol metabolism. Cholesterol is a precursor of bile acids, which are required for the absorption of cholesterol, fats, and lipophilic vitamins in the intestine [45]. Bile acids have been shown to aid in the digestion and absorption of dietary lipid [46]. Cyp46a1 binds to cholesterol and catalyzes the synthesis of the oxysterol 24 (S)-hydroxycholesterol [47]. Furthermore, Baat is the terminal enzyme for cholesterol synthesis of bile salt, which catalyzes the coupling of taurine or glycine with bile acid coenzyme, a thioester to form bile acid $\mathrm{N}$-acetamidomide [48]. A previous study found that serum cholesterol levels in mammals on a high-fat diet are high, while levels in those on a high-protein diet are low [49]. Cholesterol is the most common steroid component found in animal food. It is commonly found in animal cells [50]. We found that the Cyp46a1 gene was highly expressed in M. hoffmanni compared with M. amblycephala. Besides, the sensory system gene Tas1r1 was inferred to be positively selected in the M. hoffmanni populations. Tas1r1, a member of the Tas1r family can bind to Tas1r3 to form the umami taste receptor. Previous studies have indicated that the umami taste receptor gene T1R1 is missing in the genome of giant pandas that feed on bamboo, but multiple copies of the gene TIRI detected in the genomes of omnivorous (Oryzias latipes and Gasterosteus aculeatus), and carnivorous (Gadus morhua and Cynoglossus semilaevis) fish [11,51]. Taken together, we speculate that during its adaption to the habitat in the Pearl River, M. hoffmanni may have developed unique metabolic pathways for animal food.

\section{Conclusions}

Population genomic research provided new insights into phylogenetics, population structure, demographic history reconstruction, and feeding habits of the genus Megalobrama. The screening of selective genomic regions related to the Megalobrama diet elucidated a significant role in the environmental adaptation mechanisms of the four Megalobrama species. Importantly, this study has provided a valuable genomic resource for future genetic studies that can contribute to improvement of the Megalobrama species using genome-assisted breeding.

Supplementary Materials: The following supporting information can be downloaded at: https: //www.mdpi.com/article/10.3390/biology11020186/s1, Figure S1: Detection of stability of reference genes ( $\beta$-actin, Gapdh, and $18 \mathrm{~S} r R N A$ ). Figure S2: Gene flow analysis among different geographical Megalobrama populations. Figure S3: Ancestral area reconstruction based on BBM model (A), DEC model (B), and S-DIVA model (C) with RASP. Figure S4: Genome-wide detection of selection sweeps on chromosomes during the diet adaptation of M. amblycephala. Figure S5: Genome-wide screening for M. amblycephala (A) and M. hoffmanni (B) diet-associated selective sweeps estimating differentiation index (Fst). Figure S6: Gene Ontology (GO) analysis of candidate genes of $M$. amblycephala in MA-MH (A), MA-MS (B) and MA-MP (C) groups, and Kyoto Encyclopedia of Genes and Genomes (KEGG) analysis of these genes screened from MA-MH (a), MA-MS (b) and MA-MP (c) groups. Figure S7: Gene Ontology (GO) (A) and Kyoto Encyclopedia of Genes and Genomes (KEGG) (B and C) analyses of candidate genes in $M$. hoffmanni screened by selective sweeps inferring from the comparison between M. hoffmanni and M. amblycephala. Table S1: Mapping statistics of samples in whole genome resequencing. Table S2: Primers of the described sequences. Table S3: Comparison of stability of reference genes. Table S4: The distribution and number of samples in this study. Table S5: Data information of genome resequencing. Table S6: Variation information of samples in this study. Table S7: Candidate genes and enriched pathways in selective sweep analysis. 
Author Contributions: Conceptualization, H.L. and W.W.; Writing-review and editing, H.L., R.G., and W.W.; Visualization, J.C.; Investigation, J.C.; Writing-original draft preparation, J.C.; Formal analysis, Y.W.; Funding acquisition, W.W. All authors have read and agreed to the published version of the manuscript.

Funding: This work was supported by the National Natural Science Foundation of China (no. 31772901) and the Modern Agriculture Industry Technology System Construction Projects of China entitled as-Staple Freshwater Fishes Industry Technology System (no. CARS-45-08).

Institutional Review Board Statement: The animal experiments in this study were approved by the Institutional Animal Care and Use Committee (IACUC) of Huazhong Agricultural University (Permit number: HZAUFI-2018-008).

\section{Informed Consent Statement: Not applicable.}

Data Availability Statement: The sequence data of Megalobrama genome resequencing involved in this study have been deposited in NCBI with the BioProject accession number PRJNA756243.

Acknowledgments: We thank Z.Q. Wang, R.J. Geng, G. Cheng, and K.A. Tan for their help in sample collection.

Conflicts of Interest: The authors declare that they have no competing interests.

\section{References}

1. Du, R.; Zhang, D.; Wang, Y.; Wang, W.; Gao, Z. Cross-species amplification of microsatellites in genera Megalobrama and Parabramis. J. Genet. 2013, 92, e106-e109. [CrossRef] [PubMed]

2. Chen, J.; Guo, J.; Wang, Z.Q.; Wang, W.M. Morphological variation among the four Megalobrama species inferred by X-ray photography. Aquac. Res. 2020, 51, 3999-4010. [CrossRef]

3. Gao, Z.; Luo, W.; Liu, H.; Zeng, C.; Liu, X.; Yi, S.; Wang, W. Transcriptome analysis and SSR/SNP markers information of the blunt snout bream (Megalobrama amblycephala). PLoS ONE 2012, 7, e42637. [CrossRef] [PubMed]

4. Hu, X.; Luan, P.; Cao, C.; Li, C.; Jia, Z.; Ge, Y.; Shang, M.; Wang, S.; Meng, Z.; Tong, J.; et al. Characterization of the mitochondrial genome of Megalobrama terminalis in the Heilong River and a clearer phylogeny of the genus Megalobrama. Sci. Rep. 2019, 9, 8509. [CrossRef] [PubMed]

5. Song, W.; Zhu, D.; Lv, Y.; Wang, W. Isolation and characterization of 37 polymorphic microsatellite loci of Megalobrama hoffmanni by next-generation sequencing technology and cross-species amplification in related species. J. Genet. 2017, 96, 39-45. [CrossRef]

6. Zhang, Z.; Yu, D.; Zhai, D.; Liu, F.; Liu, H. Morphological trophic variation of the cyprinid Megalobrama pellegrini (Tchang, 1930) with different keratinized beaks from upper reaches of the Yangtze River, China. Environ. Biol. Fish 2020, 103, 115-123. [CrossRef]

7. Bai, X.; Guo, X.; Zhang, X.; Song, W.; Li, Y.; Luo, W.; Cao, X.; Wang, W. Species identification and evolutionary inference of the genera Megalobrama and Parabramis (Cyprinidae: Cultrinae) in China. Mitochondr. DNA 2015, 26, 357-366. [CrossRef]

8. He, S.; Liu, H.; Chen, Y.; Kuwahara, M.; Nakajima, T.; Zhong, Y. Molecular phylogenetic relationships of Eastern Asian Cyprinidae (pisces: Cypriniformes) inferred from cytochrome b sequences. Sci. China Ser. C 2004, 47, 130-138. [CrossRef]

9. Peng, X.; Zheng, K.; Liu, J.; Fan, Y.; Tang, C.; Xiong, S. Body size-dependent bioaccumulation, tissue distribution, and trophic and maternal transfer of phenolic endocrine-disrupting contaminants in a freshwater ecosystem. Environ. Toxicol. Chem. 2018, 37, 1811-1823. [CrossRef]

10. Xia, Y.; Li, Y.; Zhu, S.; Li, J.; Li, S.; Li, X. Individual dietary specialization reduces intraspecific competition, rather than feeding activity, in black amur bream (Megalobrama terminalis). Sci. Rep. 2020, 10, 17961. [CrossRef]

11. Liu, H.; Chen, C.; Gao, Z.; Min, J.; Gu, Y.; Jian, J.; Jiang, X.; Cai, H.; Ebersberger, I.; Xu, M.; et al. The draft genome of blunt snout bream (Megalobrama amblycephala) reveals the development of intermuscular bone and adaptation to herbivorous diet. Gigascience 2017, 6, gix039. [CrossRef]

12. Hughes, L.C.; Cardoso, Y.P.; Sommer, J.A.; Cifuentes, R.; Cuello, M.; Somoza, G.M.; González-Castro, M.; Malabarba, L.R.; Cussac, V.; Habit, E.M. Biogeography, habitat transitions and hybridization in a radiation of South American silverside fishes revealed by mitochondrial and genomic RAD data. Mol. Ecol. 2020, 29, 738-751. [CrossRef] [PubMed]

13. Zhou, X.; Guang, X.; Sun, D.; Xu, S.; Li, M.; Seim, I.; Jie, W.; Yang, L.; Zhu, Q.; Xu, J.; et al. Population genomics of finless porpoises reveal an incipient cetacean species adapted to freshwater. Nat. Commun. 2018, 9, 1276. [CrossRef] [PubMed]

14. Chen, N.; Cai, Y.; Chen, Q.; Li, R.; Wang, K.; Huang, Y.; Hu, S.; Huang, S.; Zhang, H.; Zheng, Z.; et al. Whole-genome resequencing reveals world-wide ancestry and adaptive introgression events of domesticated cattle in East Asia. Nat. Commun. 2018, 9, 2337. [CrossRef] [PubMed]

15. Zhang, Z.; Jia, Y.; Almeida, P.; Mank, J.E.; van Tuinen, M.; Wang, Q.; Jiang, Z.; Chen, Y.; Zhan, K.; Hou, S.; et al. Whole-genome resequencing reveals signatures of selection and timing of duck domestication. Gigascience 2018, 7, giy027. [CrossRef]

16. Li, H.; Durbin, R. Fast and accurate short read alignment with Burrows-Wheeler transform. Bioinformatics 2009, 25, 1754-1760. [CrossRef] 
17. Li, H.; Handsaker, B.; Wysoker, A.; Fennell, T.; Ruan, J.; Homer, N.; Marth, G.; Abecasis, G.; Durbin, R. The Sequence Alignment/Map format and SAMtools. Bioinformatics 2009, 25, 2078-2079. [CrossRef]

18. McKenna, A.; Hanna, M.; Banks, E.; Sivachenko, A.; Cibulskis, K.; Kernytsky, A.; Garimella, K.; Altshuler, D.; Gabriel, S.; Daly, M. The Genome Analysis Toolkit: A MapReduce framework for analyzing next-generation DNA sequencing data. Genome Res. 2010, 20, 1297-1303. [CrossRef]

19. De Summa, S.; Malerba, G.; Pinto, R.; Mori, A.; Mijatovic, V.; Tommasi, S. GATK hard filtering: Tunable parameters to improve variant calling for next generation sequencing targeted gene panel data. BMC Bioinform. 2017, 18, 57-65. [CrossRef]

20. Cingolani, P.; Platts, A.; Wang, L.L.; Coon, M.; Nguyen, T.; Wang, L.; Land, S.J.; Lu, X.; Ruden, D.M. A program for annotating and predicting the effects of single nucleotide polymorphisms, SnpEff: SNPs in the genome of Drosophila melanogaster strain w1118; iso-2; iso-3. Fly 2012, 6, 80-92. [CrossRef]

21. Patterson, N.; Price, A.L.; Reich, D. Population structure and eigenanalysis. PLoS Genet. 2006, 2, e190. [CrossRef] [PubMed]

22. Alexander, D.H.; Novembre, J.; Lange, K. Fast model-based estimation of ancestry in unrelated individuals. Genome Res. 2009, 19, 1655-1664. [CrossRef] [PubMed]

23. Jakobsson, M.; Rosenberg, N.A. CLUMPP: A cluster matching and permutation program for dealing with label switching and multimodality in analysis of population structure. Bioinformatics 2007, 23, 1801-1806. [CrossRef] [PubMed]

24. Rosenberg, N.A. DISTRUCT: A program for the graphical display of population structure. Mol. Ecol. Notes. 2004, 4, 137-138 [CrossRef]

25. Price, M.N.; Dehal, P.S.; Arkin, A.P. FastTree 2-Approximately maximum-likelihood trees for large alignments. PLoS ONE 2010, 5, e9490. [CrossRef]

26. Zhang, C.; Dong, S.S.; Xu, J.Y.; He, W.M.; Yang, T.L. PopLDdecay: A fast and effective tool for linkage disequilibrium decay analysis based on variant call format files. Bioinformatics 2019, 35, 1786-1788. [CrossRef]

27. Danecek, P.; Auton, A.; Abecasis, G.; Albers, C.A.; Banks, E.; DePristo, M.A.; Handsaker, R.E.; Lunter, G.; Marth, G.T.; Sherry, S.T.; et al. The variant call format and VCFtools. Bioinformatics 2011, 27, 2156-2158. [CrossRef]

28. Pickrell, J.K.; Pritchard, J.K. Inference of population splits and mixtures from genome-wide allele frequency data. PLoS Genet. 2012, 8, e1002967. [CrossRef]

29. Reddy, C.B.; Condamine, F. An Extended Maximum Likelihood Inference of Geographic Range Evolution by Dispersal, Local Extinction and Cladogenesis. Syst. Biol. 2016, 57, 4-14.

30. Yu, Y.; Blair, C.; He, X. RASP 4: Ancestral State Reconstruction Tool for Multiple Genes and Characters. Mol. Biol. Evol. 2020, 37, 604-606. [CrossRef]

31. Li, H.; Durbin, R. Inference of human population history from individual whole-genome sequences. Nature 2011, 475, 493-496. [CrossRef] [PubMed]

32. Szpiech, Z.A.; Hernandez, R.D. Selscan: An efficient multithreaded program to perform EHH-based scans for positive selection Mol. Biol. Evol. 2014, 31, 2824-2827. [CrossRef] [PubMed]

33. Huang, D.W.; Sherman, B.T.; Lempicki, R.A. Systematic and integrative analysis of large gene lists using DAVID bioinformatics resources. Nat. Protoc. 2009, 4, 44-57. [CrossRef] [PubMed]

34. Wang, P.; Li, Q. The South China Sea: Pale-Oceanography and Sedimentology; Developments in Paleoenvironmental Research; Springer: Dordrecht, The Netherlands, 2009; Volume 13, p. 506.

35. Yang, J.Q.; Hsu, K.C.; Liu, Z.Z.; Su, L.W.; Kuo, P.H.; Tang, W.Q.; Zhou, Z.C.; Liu, D.; Bao, B.L.; Lin, H.D. The population history of Garra orientalis (Teleostei: Cyprinidae) using mitochondrial DNA and microsatellite data with approximate Bayesian computation. BMC Evol. Biol. 2016, 16, 73. [CrossRef]

36. Chen, W.; Li, C.; Chen, F.; Li, Y.; Yang, J.; Li, J.; Li, X. Phylogeographic analyses of a migratory freshwater fish (Megalobrama terminalis) reveal a shallow genetic structure and pronounced effects of sea-level changes. Gene 2020, 737, 144478. [CrossRef]

37. Zheng, B.; Xu, Q.; Shen, Y. The relationship between climate change and Quaternary glacial cycles on the Qinghai-Tibetan Plateau: Review and speculation. Quatern. Int. 2002, 97, 93-101. [CrossRef]

38. Kang, B.; Deng, J.; Wu, Y.; Chen, L.; Zhang, J.; Qiu, H.; Lu, Y.; He, D. Mapping China's freshwater fishes: Diversity and biogeography. Fish Fish 2014, 15, 209-230. [CrossRef]

39. Das, A.M.; Illsinger, S.; Lücke, T.; Hartmann, H.; Ruiter, J.P.; Steuerwald, U.; Waterham, H.R.; Duran, M.; Wanders, R.J. Isolated mitochondrial long-chain ketoacyl-CoA thiolase deficiency resulting from mutations in the HADHB gene. Clin. Chem. 2006, 52, 530-534. [CrossRef]

40. Staps, P.; Rizzo, W.B.; Vaz, F.M.; Bugiani, M.; Giera, M.; Heijs, B.; Kampen, A.V.; Pras-Raves, M.L.; Breur, M.; Groen, A.; et al. Disturbed brain ether lipid metabolism and histology in Sjögren-Larsson syndrome. J. Inherit. Metab. Dis. 2020, 43, 1265-1278. [CrossRef]

41. Zhou, X.; Wang, B.; Pan, Q.; Zhang, J.; Kumar, S.; Sun, X.; Liu, Z.; Pan, H.; Lin, Y.; Liu, G.; et al. Whole-genome sequencing of the snub-nosed monkey provides insights into folivory and evolutionary history. Nat. Genet. 2014, 46, 1303-1310. [CrossRef]

42. Solden, L.M.; Hoyt, D.W.; Collins, W.B.; Plank, J.E.; Daly, R.A.; Hildebrand, E.; Beavers, T.J.; Wolfe, R.; Nicora, C.D.; Purvine, S.O.; et al. New roles in hemicellulosic sugar fermentation for the uncultivated Bacteroidetes family BS11. ISME J. 2017, 11, 691-703. [CrossRef] 
43. Ashibe, B.; Motojima, K. Fatty aldehyde dehydrogenase is up-regulated by polyunsaturated fatty acid via peroxisome proliferatoractivated receptor alpha and suppresses polyunsaturated fatty acid-induced endoplasmic reticulum stress. FEBS J. 2009, 276, 6956-6970. [CrossRef] [PubMed]

44. Krauß, S.; Vetter, W. Phytol and Phytyl Fatty Acid Esters: Occurrence, Concentrations, and Relevance. Eur. J. Lipid Sci. Tech. 2018, 120, 1700387. [CrossRef]

45. Morgan, A.E.; Mooney, K.M.; Wilkinson, S.J.; Pickles, N.A.; Mc Auley, M.T. Cholesterol metabolism: A review of how ageing disrupts the biological mechanisms responsible for its regulation. Ageing Res. Rev. 2016, 27, 108-124. [CrossRef] [PubMed]

46. Corte-Real, J.; Bohn, T. Interaction of divalent minerals with liposoluble nutrients and phytochemicals during digestion and influences on their bioavailability-A review. Food Chem. 2018, 252, 285-293. [CrossRef]

47. Lund, E.G.; Xie, C.; Kotti, T.; Turley, S.D.; Dietschy, J.M.; Russell, D.W. Knockout of the cholesterol 24-hydroxylase gene in mice reveals a brain-specific mechanism of cholesterol turnover. J. Biol. Chem. 2003, 278, 22980-22988. [CrossRef]

48. Styles, N.A.; Shonsey, E.M.; Falany, J.L.; Guidry, A.L.; Barnes, S.; Falany, C.N. Carboxy-terminal mutations of bile acid CoA:Nacyltransferase alter activity and substrate specificity. J. Lipid Res. 2016, 57, 1133-1143. [CrossRef]

49. Santos, N.; Nakamura, M.; Rio-Maior, H.; Álvares, F.; Barasona, J.Á.; Rosalino, L.M.; Santos, M.J.; Santos-Reis, M.; Ferreras, P.; Díaz-Ruiz, F.; et al. Protein metabolism and physical fitness are physiological determinants of body condition in Southern European carnivores. Sci. Rep. 2020, 10, 15755. [CrossRef]

50. Zhang, T.; Yuan, D.; Xie, J.; Lei, Y.; Li, J.; Fang, G.; Tian, L.; Liu, J.; Cui, Y.; Zhang, M.; et al. Evolution of the Cholesterol Biosynthesis Pathway in Animals. Mol. Biol. Evol. 2019, 36, 2548-2556. [CrossRef]

51. Li, R.; Fan, W.; Tian, G.; Zhu, H.; He, L.; Cai, J.; Huang, Q.; Cai, Q.; Li, B.; Bai, Y.; et al. The sequence and de novo assembly of the giant panda genome. Nature 2010, 463, 311-317. [CrossRef] 